Research Article

\title{
Brain Exercises Anywhere Anytime: A Self-Training System for Executive Function
}

\author{
Sheng-Hsiang Yu $\mathbb{D}^{1},{ }^{1}$ Wei-Lun Lin $\mathbb{D}^{1},{ }^{1}$ and Sheng-Wei Wang ${ }^{2}$ \\ ${ }^{1}$ Department of Psychology, Fo Guang University, Jiaosi 262, Taiwan \\ ${ }^{2}$ Department of Applied Informatics, Fo Guang University, Jiaosi 262, Taiwan \\ Correspondence should be addressed to Wei-Lun Lin; wllin.fgu@gmail.com
}

Received 26 September 2019; Revised 12 November 2020; Accepted 31 January 2021; Published 12 February 2021

Academic Editor: Alessandro Bazzi

Copyright (c) 2021 Sheng-Hsiang Yu et al. This is an open access article distributed under the Creative Commons Attribution License, which permits unrestricted use, distribution, and reproduction in any medium, provided the original work is properly cited.

\begin{abstract}
Smartphones are ideal for tracking individuals' behavioral responses. Traditional laboratory-based psychological trials aimed at training cognitive functions face a problem when participants cannot finish their tasks within a scheduled time. In this paper, we developed a self-training system that helps people to enhance their cognitive functions through mental exercises. The system utilizes the advantages of mobile computing and Internet connection. Participants can finish their mental exercises no matter where they are and can send the results back to the data server. Administrators can track all participants' status on a web-based system. This study investigates in situ the 20 -session use of a mobile application. The results reveal that the users significantly enhanced their level of executive functions. The dropout rate was very small. This application, which uses a cloud data server, makes it more efficient and cost-effective in conducting psychological training programs. The proposed system provides a basic smartphone-based system for psychological trials and can be further improved by combining other techniques such as speech recognition and data-mining techniques.
\end{abstract}

\section{Introduction}

With the recent advances in wireless network technology, smartphones are playing an increasingly important role in humans' daily lives $[1,2]$. People can use smartphones to communicate with each other via the Internet. Many interesting applications such as games and e-book readers can be installed on a smartphone. The mobile computation capability of smartphones makes them powerful tools and provides researchers from different academic disciplines with a new opportunity to collect varying data from more users worldwide. Smartphones are ideal for tracking individuals' psychological and behavioral responses [3]. For example, smartphones can be used to track one's sleep behaviors [4] or to help users with practicing mindfulness skills in their daily life [5]. The smartphone is also an ideal platform to plan and implement information systems designed for learning or training [6].

This paper develops a new cognitive-training protocol. Traditionally, participants in psychological trials must finish their tasks in a laboratory at a scheduled time. A complete experimental trial consists of several tasks that must be done on different days. However, many participants will not finish their experiments because they cannot get to the laboratory at their scheduled time due to weather, traffic, or other personal reasons. In social science research, this attrition issue (i.e., subject loss or dropout) may limit a study's validity and increase the costs of collecting sufficient data $[7,8]$.

An efficient solution to overcome this problem is to develop a smartphone-based application for participants so that the participants can finish their tasks anywhere at any prespecified time. In this paper, we propose a new self-training system, which can be applied to enhance individuals' performance in high-order cognitive functions, called executive functions (EFs) $[9,10]$. This self-training system allows participants to do reading and arithmetic exercises on their smartphones at any place, and the results will be sent back to the data server. Baker et al. [11] found that playing computer learning games 
can result in increased cortical activation. The results of brain imaging studies have revealed that reading sentences aloud [12,13] and doing simple arithmetic operations $[14,15]$ greatly activate individuals' prefrontal cortex. Researchers have also indicated that these two tasks involve cognitive processes requiring executive functioning. During a training session, some participants may miss some exercises. The administrator should be aware of this situation, and the participant needs to be notified. Therefore, the proposed self-training system has the following three goals:

(1) The participants must be able to do mental exercises (reading and arithmetic) on a smartphone by themselves, no matter where they are

(2) The administrators must be able to monitor the response profile of each participant, including their voices when reading and the results of their arithmetic exercises

(3) The system must automatically remind participants, to reduce the attrition rate

Different from most applications installed on smartphones, the applications for psychological experiments must meet some special requirements. First, the privacy of user information and their experimental results must be strictly protected, both on smartphones and in data servers. Data transmission between smartphones and the data server should be secured. Second, the user interface must meet the guidelines of psychological experiments specified in $[7,8]$.

To meet the requirements and goals for the expected self-training system, we designed and developed an Android application and a backend system. The application can be installed on all Android smartphones with Internet access. Every day when a participant finishes their exercise, the data of the exercise results will be uploaded to the data server. The experiment's administrator can track the results from all of the experiment's participants. Therefore, if a participant misses some exercises or does not concentrate on doing the exercise, the administrator can notify the participant. The authors expect that after the experiments are completed, most participants will be able to finish the training and most of the result data will be valid and useful. Moreover, after accepting training by our system, the participants are expected to improve in terms of EF.

The proposed system provides a basic system for psychological trials. In the future, some techniques can be added to the system for further use. For example, the trials can be adopted according to the participants' answers. The system would then become an adaptive trial system. Another example is that the experimental results can be further analyzed based on data-mining techniques, with which the results of trials can be predicted and some interesting results can be obtained.

The rest of this paper is organized as follows. We define EF and discuss previous works on smartphone-based applications in the next section. This system's requirements are listed in Section 3. Section 4 describes the system's development and implementation steps. Some statistics and results are shown in Section 5. Finally, some concluding remarks will be given.

\section{Literature Review}

In this section, we first introduce the definition and recent studies of the executive functions. Then, the applications installed on smartphones for psychological experiments are reviewed.

2.1. The Executive Functions. The EFs can be defined as cognitive processes that involve the abilities to initiate, plan, engage in goal-directed behaviors, and monitor the consequences of one's actions $[9,10]$. Theories and accumulating evidence indicate close relationships between EFs and emotional regulation [16-18] attitude change, and thinking and creative abilities [19]. EFs are essential for problem-solving and environmental adaptation. Thus, the investigations into EFs have promising value for positive psychology, which is aimed at improving people's positive emotions and attitudes and enhancing their strengths and potentials. Recent neurocognitive studies generally indicate that EFs are located in humans' prefrontal cortex [20-22]. Notably, researchers have discovered the plasticity of this area and developed training programs to activate the prefrontal cortex and hence improve EFs $[23,24]$. Therefore, developing protocols to improve fundamental cognitive functions and, hence, emotion regulation and problem-solving abilities would be valuable.

Recent studies have also demonstrated positive correlations between EFs and students' academic achievement. For instance, researchers have adopted the Trail-Making Test [25] to measure adolescents' shifting ability and found that their performance was positively correlated with their mathematics grades [26]. Researchers have used the operation span task [27] to measure elementary school students' updating function and found that updating function could positively predict students' English, mathematics, and science class performances [28]. Best, Miller, and Naglieri [29] examined the complex EFs of students aged 5 to 17 (the subtests involved planning and monitoring from the Cognitive Assessment System (CAS); [30]) and found a steady strength in the correlations with students' math and reading achievement across ages.

The reviews of Gruzelier [31] indicated that neurofeedback (NF) training can improve cognitive performance in healthy adults. NF is effective in enhancing individuals' attention [32], sensory sensitivity [33], and working memory $[34,35]$. Despite these positive results of NF, the application of NF is still limited due to the large costs involved. Basically, $\mathrm{NF}$ is a lab-based method, making it time-consuming and location-restricted. It usually consists of eight to 10 sessions, each taking from half an hour to one hour $[35,36]$. Moreover, a certain percentage of people (around $10 \%$ across different studies) are unable to learn to self-regulate through NF, who are sometimes called nonresponders. Some trainees may drop out due to fatigue or because they 
cannot attend the training sessions as scheduled for personal reasons.

Behavioral training is another evidenced EF-enhancing approach. Researchers have indicated that regular behavioral activities such as solving simple arithmetic problems and reading aloud-so-called "mental exercise"-can effectively improve EFs in both elderly and young adults [37-39]. In short, behavioral training might be a simple and convenient means of enhancing individuals' EFs.

Compared with NF, behavioral training is more convenient, instrument-free, easily customized, and selfadministrated. These characteristics of behavioral training provide great opportunities in mobile computing to develop a self-administering training tool for people who want to improve their fundamental cognitive functions. Thus, the major purpose of the present study is to develop an EF-training tool that will enable participants to do "mental exercise" (to read aloud and solve arithmetic problems) by themselves on mobile devices. The participants' EF measurements in the posttraining stage (T2) are expected to improve in comparison with their performance in the pretraining stage (T1).

2.2. Smartphone-Based Applications for Psychological and Behavioral Studies. Some researchers have employed mobile-phone-based tools to track and share behavior data [40], such as stress and sleep behavior [4, 41,42], mindfulness and worry [5, 43], movement behaviors [44], and interpersonal behaviors [45-47]. Some recent review studies demonstrated the efficacy of smartphone-based applications in intervention contexts $[48,49]$.

For example, Yu et al. [47] developed a mediation tool for improving the interaction between depressed individuals and caregivers. The mobile care mediation tool allows depressed individuals and their specific caregivers to share their moods and current states. Not only did the depressed individuals reduce their reassurance-seeking behaviors, but the caregivers' burden was also reduced. Meanwhile, the users also reported high satisfaction with their relationship. Additionally, psychologists can explore the interpersonal and emotional factors of depressed individuals and caregivers with the assistance of technology. Cahittro and Vianello [5, 43] developed a mobile mindfulness app (AEON) to assess participants' mindfulness and user experience with the app. Several participants in this study experienced decentering from their worries when using the app. Howells et al. [50] also demonstrated the viability of the smartphone-based mindfulness intervention which could effectively enhance individuals' well-being.

Nouchi et al. [51] asked participants to play a commercial brain-training video game (Brain Age, published by Nintendo Co. Ltd., including arithmetic and reading activities) on a portable console (Nintendo DSi) at home over 4 weeks, with at least 4 training days each week. The results showed that Brain Age improved EFs, working memory, and processing speed in young adults. The active control group, which played a puzzle game (Tetris) instead, can make improvements in their visuospatial abilities alone but not in
EFs. The authors concluded that the Brain Age game might be a convenient way to improve EFs. Nevertheless, it is still unclear which activities in this complicated video game can enhance EFs. In fact, the Brain Age game comprises activities other than solving arithmetic problems and reading aloud. Based on the evidence of previous studies $[39,52,53]$, the cognitive-function-boosting effects might be due to solving arithmetic problems and reading aloud specifically.

Since the Nintendo DSi does not have the functions that record participants' data (such as playing time and scores), a stopwatch was provided for participants to record their game performance in a training journal. The experimenters had to check whether the participant-reported practice times in the training journal matched the actual practice times on the stopwatch.

\section{System Requirements}

In this section, we first precisely define the terminologies used in this paper. The system requirements of the smartphone-based application and backend system are then described.

3.1. Terminology. In this paper, we use the following terminology to describe the entire psychological experiment system. Users of this system can be classified into two categories: administrator and participant. The participant in the system is the subject of the experiment while the administrator conducts the experiment. A participant will do a 15-minute exercise every day. An exercise includes both reading articles and arithmetic operations. The multiple exercises completed by each participant are called a session, and the set of sessions conducted by a single administrator is called a training project.

3.2. Detailed System Requirements. The proposed system consists of two main parts: a smartphone-based application and a backend system. The detailed requirements of the two parts of the system are described in the following sections.

3.3. Smartphone-Based Application. The training exercises are completed via smartphone-based applications. Results of data are stored in the smartphone and are sent to the backend system via a secure connection. The detailed requirements of the smartphone-based application are as follows:

(i) Because the protection of personal information is the most important issue in this system, login and password checking is required. By using an accountmanagement procedure, the application can be used by multiple participants on a single device.

(ii) For each person, only one exercise can be done per day. When the number of exercises reaches 20 , the participant will have completed their training session. 
(iii) In each exercise, the minimum reading exercise time is set to 7 minutes, and the residual time is used for the arithmetic exercise. The exercise will stop when 15 minutes is up.

(iv) For each person, difficulty levels are set separately for the reading and arithmetic exercises. For each participant, the difficulty levels of the reading and arithmetic exercises are set in advance.

The reading exercises will include different articles at different difficulty levels. The articles are manually classified into three levels by the administrator. The three difficulty levels are set based on the materials used in formal education for different age groups [39]. Each level comprises 80 articles, which are randomly selected for reading. No article will be presented twice to a participant throughout the training session.

For arithmetic exercises, the problems are first divided into the following three levels.

(i) Level 1: Only integer addition, subtraction, and multiplication problems can be generated. The addition and multiplication problems are carry-save problems. The subtraction problems require no borrowing.

(ii) Level 2: The problems are integer addition, subtraction, and multiplication problems without any constraints.

(iii) Level 3: The problems are Level 2 problems as well as the integer division problems. For the division problems, the dividends are all divisible.

The principles used to generate the arithmetic problems are as follows:

(i) Level EASY: 50\% Level 1 and 50\% Level 2 problems

(ii) Level NORMAL: 20\% Level 1 and 80\% Level 2 problems

(iii) Level HARD: 20\% Level 2 and 80\% Level 3 problems

3.4. Web-Based Backend System. The backend system is used to manage participants and collect training results. The requirements of the backend system are as follows:

(i) For privacy protection, the backend system is secured with a login/logout function

(ii) The administrator of a training project can add, delete, and edit a participant's information, including their username and password

(iii) The system can receive voice files from smartphones, and the voice files can be played through a website

(iv) Each participant's experimental results can be shown on a web page

\section{System Development and Implementation}

A smartphone-based application with a web-based backend system in the cloud was developed to meet the requirements specified in Section 3. This section provides an overview of the system architecture. The design of the smartphone-based application and its backend system was then developed. Finally, the integration was applied to the system.

4.1. System Architecture. A smartphone-based application can be developed for the Android or iOS platforms. In this paper, we developed the application on the Android 4.1 platform. The backend system is a web-based application with database support. We used the OpenShift cloud platform [54], which supports PaaS services. The web-based application was developed using the PHP language, and the database system utilizes MySQL 5.1 with the phpMyAdmin management tool.

4.2. Database Design. A database with several tables was designed to meet the web-based system's requirements. Figure 1 shows the system's entity-relation model (ER model). The database contains six entities. The ADMINISTRATOR table records the account and password of each administrator. Different administrators are responsible for different sets of participants and deal with different training projects. The PARTICIPANT table contains the information about each participant, and the Admin ID field is used to associate each participant with their administrator. Two difficulty levels_namely, reading level and arithmetic level-are used to select the articles for reading and to generate arithmetic problems. For security reasons, the password fields in ADMINISTRATOR and PARTICIPANT are the encrypted strings after the MD5 hash function is applied to the original password strings.

When a participant completes an exercise, the results will be uploaded to the system and stored in three tables. The EXERCISE table contains who completed the exercise and when. Detailed results are stored in the READING DETAIL and ARITHMETIC DETAIL tables, which are associated with a record in the EXERCISE table with EXERCISE ID.

The articles are stored in the ARTICLE table. Each record contains a unique ARTICLE ID, the article's contents, and its difficulty level. The arithmetic problems do not need to be stored in the database because the problems are generated online according to the rules specified in Section 3.2 .

4.3. Design of the Smartphone-Based Application. The functions in the smartphone application are classified into four parts: member login/logout, the reading exercise, the arithmetic exercise, and uploading of the records. The implementation and participant-interface design are described hereinafter.

4.3.1. Member Login/Logout Function. First, we describe the login function. Since this self-training system is not a public system, only the participants added by the system administrator can use the system. The personal information, login account, and password of each participant are manually added to the backend system. Figure 2 shows the participant 


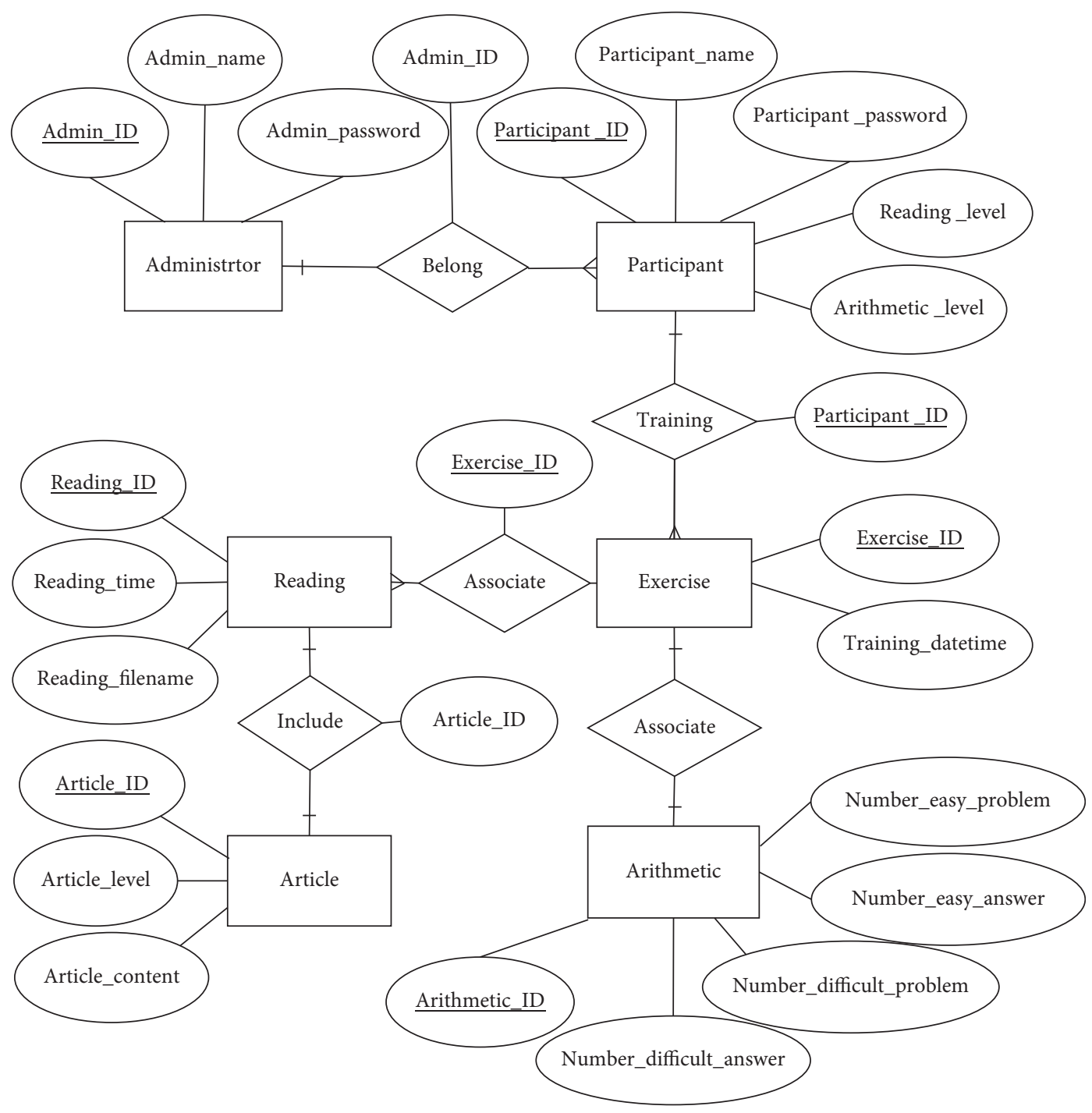

Figure 1: Entity-relation model.

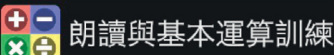

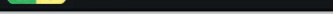

請輸入帳號密碼

\section{請在此輸入帳號 Account \\ 請在此輸入密碼 Password}

\section{登入}

\section{Login Button}

Figure 2: Login interface. 
interface of the login page, which is also the first page of this application.

In order to guarantee security and privacy, the system uses a private account and corresponding password to authorize and authenticate those who are permitted to use this system for self-training. When a participant enters their account with a password and presses the login button, the application will start a network connection to the backend system. If the account exists and the password is correct, the participant can then use the system to view their exercise history. Otherwise, the application will show an error message according to the error code returned from the backend system. In order to prevent the plain text password from being intercepted by others, the password string is hashed by the MD5 method in the mobile device. When the login button is pressed, the password string entered by the user is first hashed and the encrypted string is then sent out by HTTP connection to the server. When the server receives the string, it compares the received string with that in the database. If the two strings are identical, the user is permitted to enter the system for training. Otherwise, the system sends an error message to the mobile device.

Figure 3 shows the page when a participant successfully logs into the system. This page shows the participant's history records. Each record shows the exercise date, the number of articles read, and the number of arithmetic problems along with the number of correct answers. This page also has a button to start exercises. Because the system limits each participant to exercise at most once per day, this page will check if the participant has completed the training that day or not. If the participant has completed the training for the day, the button will be invisible. In addition, the button will also be invisible if the participant has completed all of their exercises (20 exercises). Otherwise, the participant can press the training button to start their exercise for the day.

Figure 4 shows the pseudocode of the algorithm used to process a training session. When the participant presses the Start Training button, the reading exercise will be shown first. Figure 5 shows the instructions used to teach the participant how the reading exercise will proceed. The training will begin when the participant presses the Start button. The initialization steps of the reading exercise are as follows:

(1) An article that the participant has not read before will be randomly selected from the set of articles with the same difficulty level

(2) The microphone of the smartphone is turned on to record the participant's voice

(3) A text file is created to store the training records and prepared for uploading to the cloud database

Figure 6 shows the participant interface for the reading exercise. Each page shows a sentence, limited to only one line. When the participant finishes reading the sentence, they should press the Next button to get to the next sentence. To separate the articles into several sentences, we insert some special characters into the articles and a finish symbol to indicate that there is no next sentence in the article.
Based on the system requirements, the reading exercises must last at least 7 minutes. If the reading training lasts less than 7 minutes, another article that has not been read before will be selected, and the reading exercise will continue until the article is completely read. At this time, the file that records the participant's voice will be closed, and a new voice file will be opened. The file names will be recorded for uploading to the cloud file system. Figure 6 shows the participant interface showing that the next article is ready to begin. Note that the time between the two articles is not added to the total training time. If the reading exercise for an article is finished and the total reading time is greater than or equal to 7 minutes, then the training is switched to arithmetic exercises. Figure 7 shows the interface showing that the reading is finished and the instructions for the arithmetic exercise.

Figure 8 shows the application's arithmetic exercise interface. The problems are generated according to the participant's difficulty level. During the exercise, the number of generated problems and the number of correct answers will be shown on the screen. The problems will be generated repeatedly until the total training time approaches 15 minutes. When the training time equals 15 minutes, the training exercise for the day will be complete, and the results will be uploaded to the cloud database. Figure 9 shows the final page stating that an exercise has been completed. To minimize the bandwidth consumption of the network connection, the results are encoded into a string in JSON format. The voice files are uploaded at the same time. If a network connection is unavailable when the exercise is completed, the results will be temporarily stored in a text file. When the network connection has been resumed, the results and the voice files will be uploaded automatically, and all files will then be removed from the smartphone. In addition, a summary of the training will also be shown after an exercise has been completed.

4.4. Design of the Web-Based Backend System. Since a backend system is required to provide stable services for the application, we choose the OpenShift Cloud Service, a PaaS service, for our system. The backend system is a web-based application using the MySQL database system. We used the PHP language to develop the backend system.

The services provided by the backend system can be divided into two parts: data processing and participant administration. The data-processing functions include parsing the JSON string from the mobile application and inserting it into the database. The participant administration includes member management and monitoring of the participants' training status.

When an exercise is completed, a JSON string and a set of voice files will be uploaded. The JSON string contains the reading and arithmetic results of the training. The backend system first parses the JSON string and then inserts the results into the corresponding tables. Voice files are also stored in a specified directory with a new file name. The user ID, article ID, and start time of the reading exercise will all be reformatted with a new file name. 
$\stackrel{+\ominus}{\times \bullet}$ 朗讀與基本運算訓練

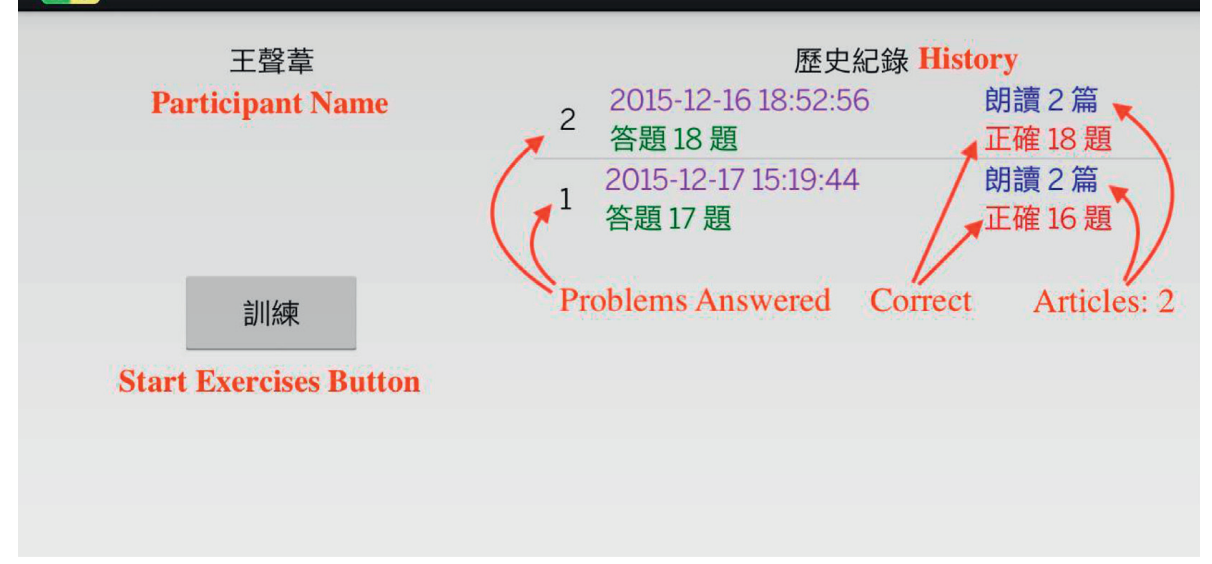

Figure 3: Personal history.

Algorithm 1 Training Algorithm on Smartphone
(1) Show the instructions for reading training
(2) Start Reading Timer
(3) while (Reading_Timer $<7$ minutes) do
(4) Open microphone and recorde the voice

(5) Select an article which has not

been read

(6) Save the voice file

(7) end while

(8) Stop Reading_Timer

(9) Show the instructions for arithmetic training

(10) Start Arithmetic_Timer

(11) Set total_questions to 0

(12) Set correct_answers to 0

(13) while (Reading_Timer + Arithmetic_Timer $<15$ minutes)

do

(14) Generate an arithmetic

problem

(15) total_questions = total

questions +1

(16) User answers the question

(17) if (Correct Answered) then

(18) correct_answers $=$ correct_answers +1

(19) end if

(20) end while

(21) Stop Arithmetic_Timer

(22) Upload the voice files and arithmetic results

FIgURE 4: The algorithm used to process a training session.

When an administrator logs into the backend system, they can get the list of participants as well as the number of training exercises completed. Figure 10 shows the interface of the participant list. The participants' accounts (email) can be clicked on, and the page will be switched to the details of their training history. Figure 11 shows the list of training exercises with detailed information. The number of completed reading exercises and their corresponding voice files are also listed, alongside the results of the participants' arithmetic exercises.

\section{Results and Discussion}

The self-training system is proposed to enhance EFs. In this section, we first describe the uniqueness of the proposed system. Some statistics of the proposed system show that the percentage 
$\oplus \ominus$
$x \Theta$ 朗讀與基本運算訓練 王聲蔁

\begin{tabular}{|c|}
\hline 指導語 \\
\hline 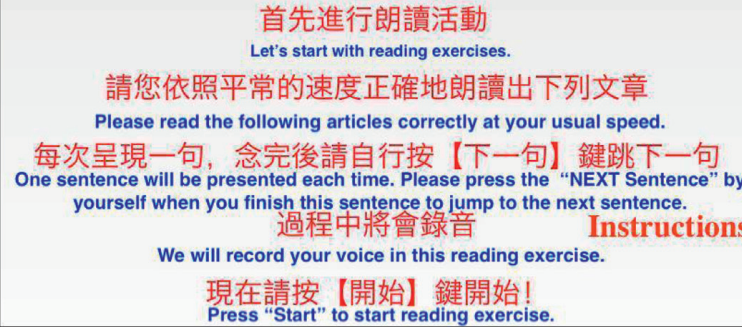 \\
\hline
\end{tabular}

Start Button

Figure 5: Instructions on how to do the reading exercise.

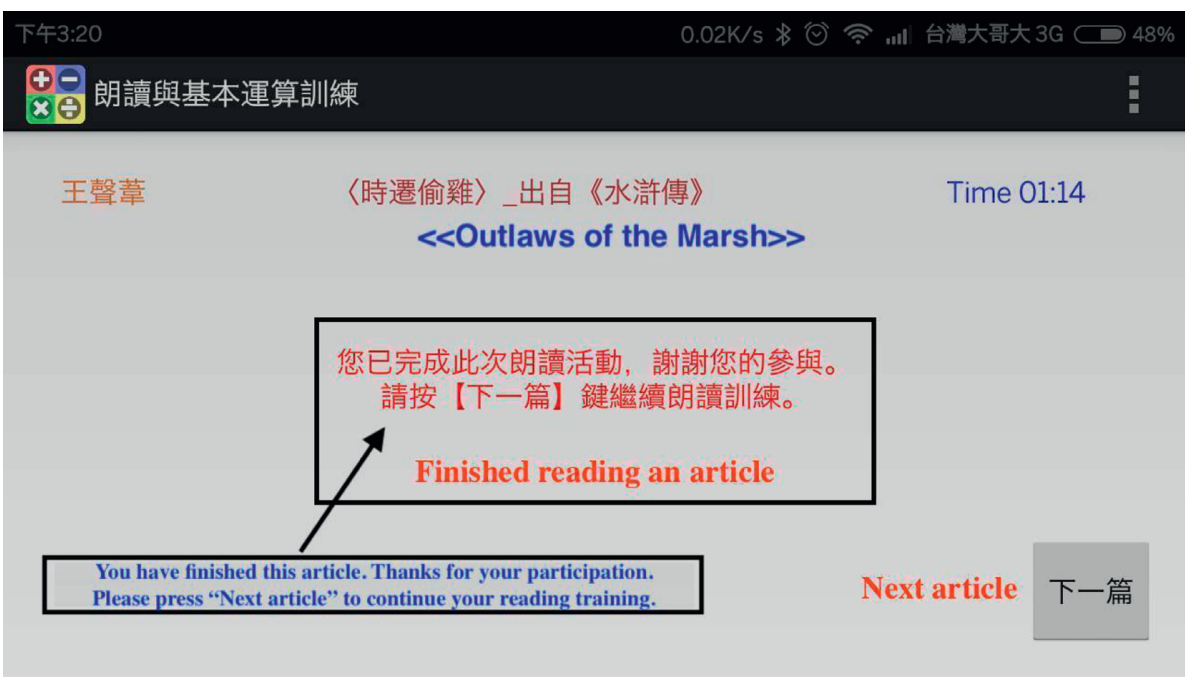

FIgURE 6: The user interface of reading exercise.

您已完成此次朗讀活動, 謝謝您的參與

You have finished the reading exercise. Thanks for your participation. 接下來要進行數學運算活動

每次呈現一則數學題, 請一定要用心算解題

再用數字鍵盤回答答案, 並按Enter鍵跳下一題 現在請按【開始】鍵開始!

Let's do the next training activity: the arithmetic exercise. One arithmetic problem will be presented each time. Use the numeric keypad to input your answer. Please press "Enter" to jump to the next problem.

FIgURE 7: Reading of an article is completed. 


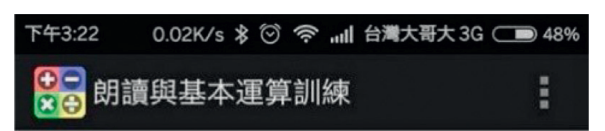

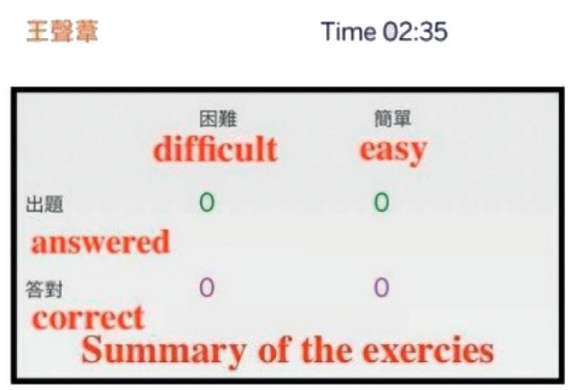

$$
684 \div 2=342
$$

The problem

\begin{tabular}{|l|c|c|c|}
\hline 1 & 2 ABC & 3 DEF & - \\
\hline 4 GHI & 5 JKL & 6 MNO & - \\
\hline 7 PQRS & 8 TUV & 9 WXYZ & X \\
\hline$* \#($ & $0+$ & English & 完成 \\
\hline
\end{tabular}

(a)

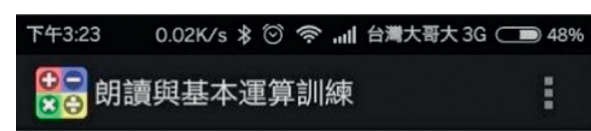

主聲蕃

Time 03:14

\begin{tabular}{|c|c|c|}
\hline & difficult & $\begin{array}{c}\text { 簡單 } \\
\text { easy }\end{array}$ \\
\hline $\begin{array}{l}\text { 出題 } \\
\text { answered }\end{array}$ & 1 & 7 \\
\hline correct & 1 & 6 \\
\hline
\end{tabular}

$955-318=$

FIgURE 8: Participant interface of arithmetic exercise.

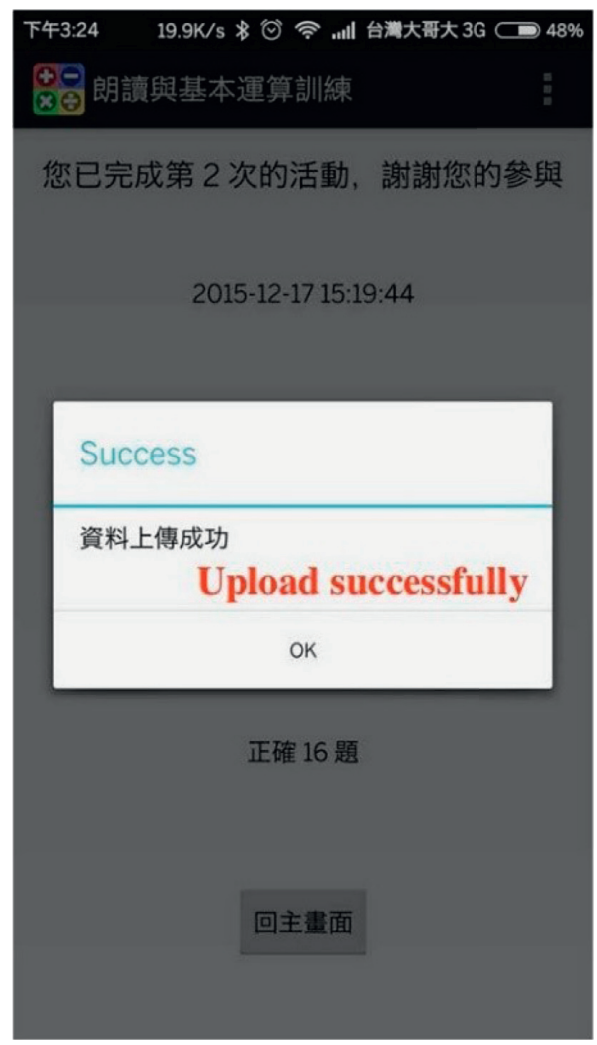

(a)

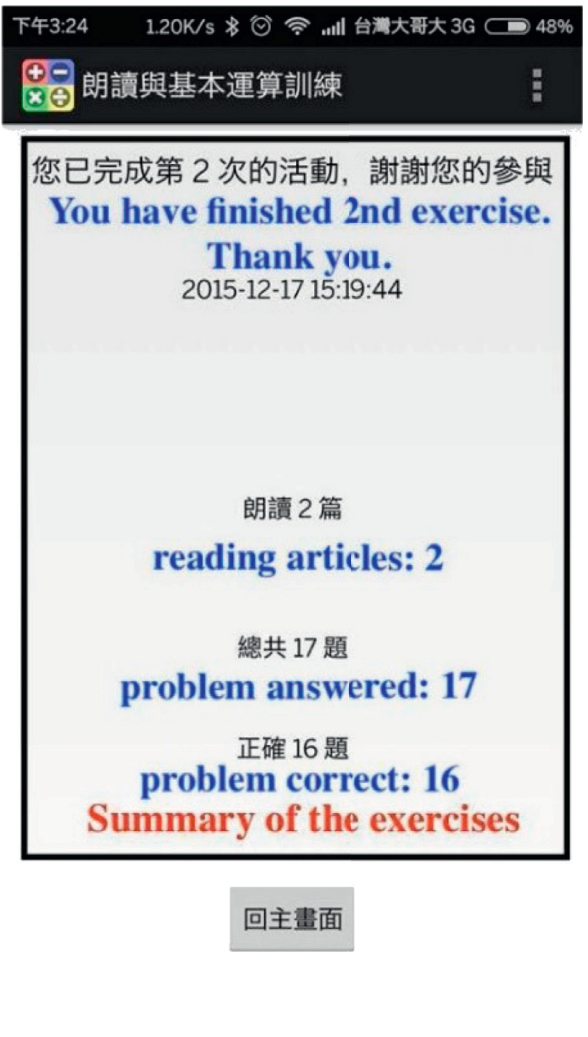

(b)

FIGURE 9: Upload the record to the cloud system and the summary of the results. 


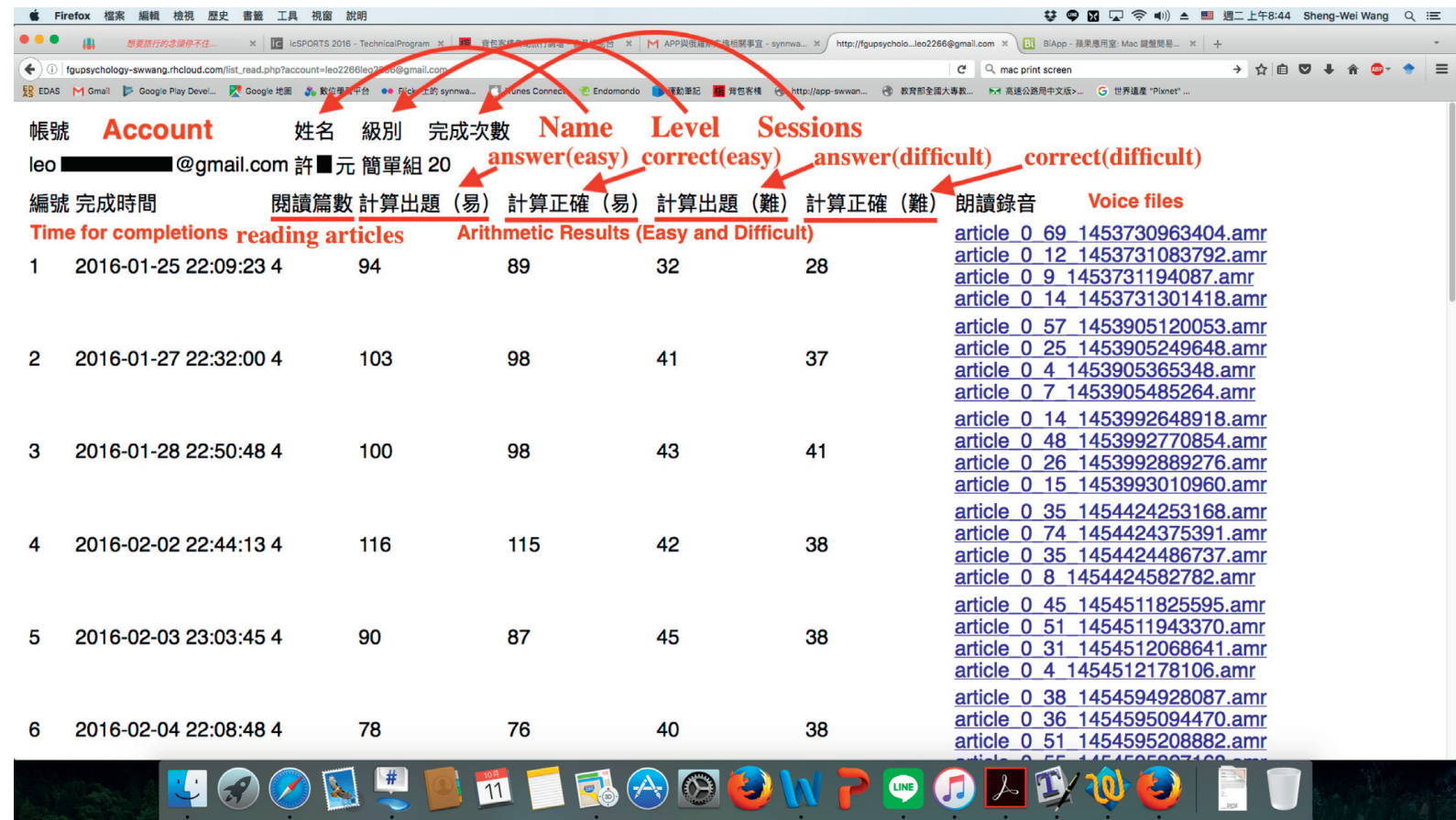

FIgURE 10: Summaries of participants in a project.

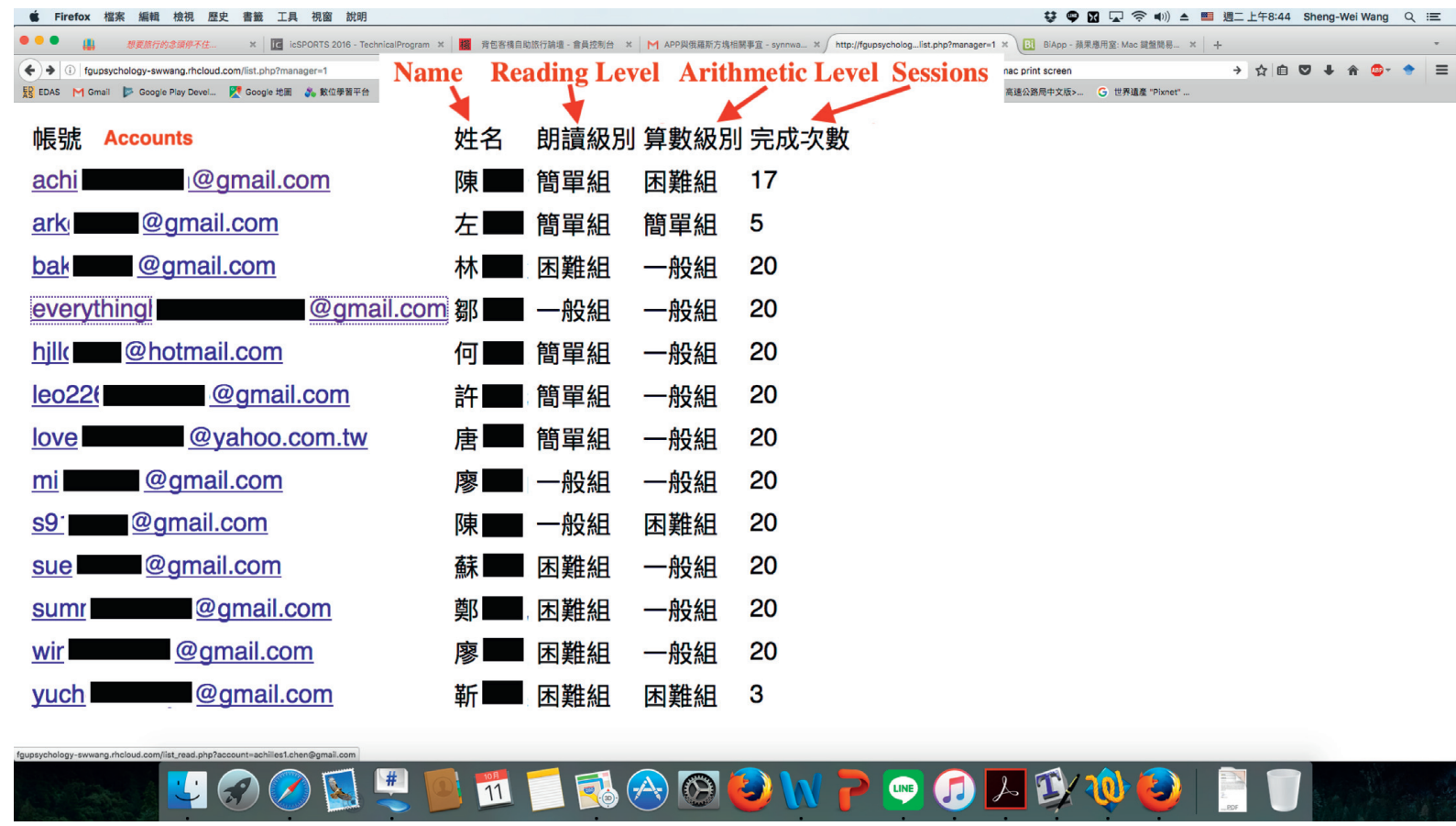

FIgURE 11: Detailed results of a participant. 
of lost participants decreases by using the system. Finally, the effectiveness of the system shows that the system is useful for enhancing the executive function of a participant.

5.1. Uniqueness of the Proposed System. This system has some advantages compared to other EF-training methods used in other related works. Compared with traditional paper-based cognition training (e.g., [39]), this proposed architecture can provide adaptive and individualized training based on the trainee's cognitive ability. Moreover, this architecture is also extensible. Different content and parameters of the training sessions such as frequency and duration can vary in different trials.

In addition, the proposed system has the following advantages over the Nintendo tool used by Niendam et al. [21].

(1) We use Android smartphones as the devices for the training projects because these are much more widely used than the Nintendo DSi handheld console is.

(2) The administrator can manually set the difficulty level according to each participant's capabilities.

(3) Our system can record and upload the participants' voices when reading so that the administrator can monitor whether the participants are doing the tasks properly.

(4) The system will record the reading and arithmetic results after each exercise so that each participant's progress can be tracked.

5.2. Dropout Rates. A participant is considered a dropout if they quit the training project without finishing their training session (20 exercises). A lower dropout rate results in lower costs of collecting enough data. Table 1 shows the dropout rates from the training projects using the proposed system. We have conducted three experimental projects using the proposed self-training system. The dropout rates were relatively low. For example, Uchida and Kawashima [39] had a $17.74 \%(11 / 62)$ dropout rate from their traditional paperbased cognitive training, which involved solving arithmetic problems and reading. The dropout rate for a 20 -session NF training performed with depressed college students was $13.33 \%(4 / 30)$ [55]. The results reveal that using the proposed system can lower the dropout rate, as compared to using traditional laboratory-based experimental methods. Thus, using a smartphone-based application with a cloud data server is an efficient and cost-effective way to conduct psychological experiments.

5.3. An Efficient and Practical Means of Enhancing EFs. We have developed a ubiquitous self-training system that provides an efficient and convenient means of enhancing one's EFs. This self-training system is a much more practical and economical solution than the neurofeedback approaches to improve individuals' EFs, which were reviewed above. A recent review showed that NF protocols designed to enhance
TABLE 1: Dropout rates of the training projects.

\begin{tabular}{lccc}
\hline Projects & Participants & Dropouts & Dropout rate $(\%)$ \\
\hline Project 1 & 20 & 3 & 15.00 \\
Project 2 & 17 & 0 & 0.00 \\
Project 3 & 10 & 1 & 10.00 \\
Total & 47 & 4 & 8.52 \\
\hline
\end{tabular}

one's cognitive performance usually require 15-40 training sessions, with each session taking from half an hour to one hour [56]. The mean number of NF sessions in an attention deficit and hyperactivity disorder (ADHD) intervention ranges from 20 to 50 [57]. In order to examine the effects of utilizing our EF-training tool, we recruited 17 undergraduate students to use this system for 20 training sessions, each lasting 15 minutes. All of the participants finished their tasks. Based on the feedback collected from the 17 participants who used this system, their motivations were maintained over time, rather than decaying. As Table 2 shows, the participants' positive motivations (self-rated concentration and interest in using the application) and negative motivation (feeling tired) showed no statistically significant differences between the two time-point assessments.

To examine the proposed system's enhancing effects on EFs, three cognitive tasks were used to evaluate the 17 participants' EFs pre- and posttraining. The operation span task [27], the number-letter task [58], and the latent inhibition task [59] are representative tasks for measuring the three components of EFs: updating, switching, and inhibition [60]. In the operation span task, participants were asked to verify the correctness of arithmetic equations and memorize words simultaneously, hence manipulating and monitoring their EF content. Their performance could be scored from level 2 to 7 . In the number-letter task, pairs of stimuli consisting of a number and a letter were presented consecutively on the computer screen. The participants were first asked to judge whether the number was odd or even and were then asked to switch to another judging rule, according to which the participants had to judge whether the letter was a capital letter or lowercase. The response time (RT) difference between switching and nonswitching trials is considered a switching cost, with smaller values being better. In the latent inhibition task, participants were asked to respond to irregular geometric shapes presented on the computer screen. Each trial included one target and 19 distractors (with the same shape). After 64 trials, the distractors became a target, which usually increased the RTs. The larger the extent of the increase of the RTs, the more the distractor is being inhibited. Each participant's performances on these three tasks were standardized separately and summed as a composite index of EFs.

The pre- and posttest comparisons of the EFs indices are shown in Figure 12. Our participants' performances in every aspect of EFs improved after utilizing our EF-training tool, especially their switching ability $(t=4.755, p<0.001)$. Overall, the participants' EFs significantly enhanced from pre- to posttraining $(t=2.501, p<0.05)$. 
TABLE 2: User experiences (UXs) of the self-training system.

\begin{tabular}{lccccr}
\hline UXs & \multicolumn{2}{c}{$\begin{array}{c}\text { During- } \\
\text { training }\end{array}$} & \multicolumn{2}{c}{$\begin{array}{c}\text { After-training } \\
\text { (4th week) }\end{array}$} & T-test \\
& \multicolumn{2}{c}{ (1st week) } & & & \\
& Mean & Std. & Mean & Std. & \\
\hline Concentration & 3.79 & 0.58 & 3.36 & 0.75 & -1.71 \\
Interest & 3.36 & 0.75 & 2.93 & 0.73 & -1.58 \\
Tired & 2.79 & 1.05 & 2.71 & 1.07 & -0.32 \\
\hline
\end{tabular}

*The scores of UXs range from 1 to 5 .

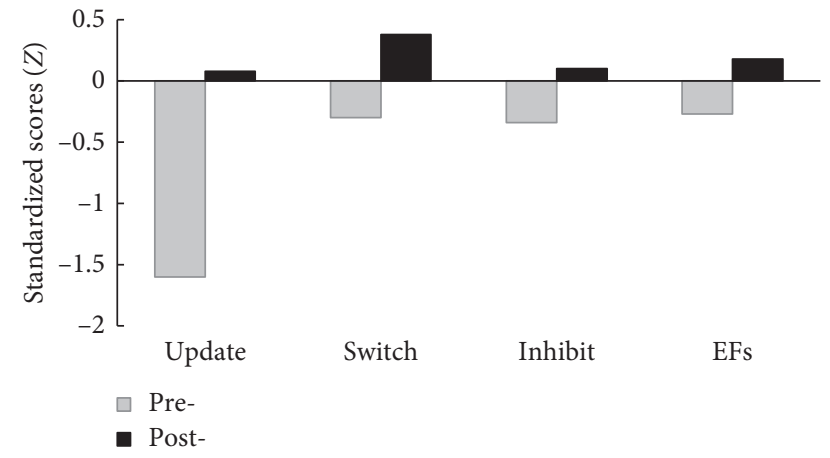

FIgURE 12: The comparison of the performance in different EF tasks.

\section{Conclusions}

In this paper, we have proposed a ubiquitous self-training system for enhancing EF. The system consists of a smartphone-based application and a backend system for a data server. Compared with previous research that used Nintendo DSi handheld consoles, the proposed system adds some useful functions, which can help the administrator to trace each participant's training. Using the system can decrease the number of dropouts, which can considerably reduce the cost of psychological experiments. Also, the participants' motivation can be maintained, which is vital for improving voluntary participation in research involving human subjects. However, given that the sample size was small in this exploratory study, a more appropriate conclusion would have been to call for further study with a larger sample. In addition, with authentication technologies such as fingerprint and face recognition that can be easily implemented in mobile apps in the future, these technologies can be further applied to guarantee users' training processes and hence the efficacy of the training results.

Given EFs are essential for adaptation and for coping with challenging environments, means that can effectively enhance EFs have great potential in improving psychological health and well-being. The undergraduate students who used our proposed self-training system for five weeks showed significant improvement in different aspects of the EFs. This ubiquitous system can provide a simple and effective way to enhance one's core cognitive performance. Promising practical value is expected in education and clinical fields. For example, it would be worthwhile to apply this system to depressed patients $[61,62]$ or to elderly participants $[63,64]$, whose EFs are known to be impaired. Changes in human behaviors attract psychologists' attention. The high accessibility of smartphones provides researchers with an opportunity to design and conduct psychological studies. Additionally, the user experiences using this kind of self-training system can contribute valuable feedback to application designers with regard to human-machine interfaces. In the future, this system can be combined with other techniques such as speech recognition and datamining techniques for other psychological trials.

\section{Data Availability}

The data used to support the findings of this study are available from the corresponding author upon request.

\section{Conflicts of Interest}

The authors declare that there are no conflicts of interest regarding the publication of this paper.

\section{Acknowledgments}

This research was supported by the Ministry of Science and Technology, Taiwan, under grants MOST104-2221- E-431-001, MOST104-2410-H-431-003, MOST104-2410- H-431-006, MOST105-2632-E-431-001, and MOST105- 2410-H-431-020.

\section{References}

[1] M. Ghahramani, M. Zhou, and C. T. Hon, "Mobile phone data analysis: a spatial exploration toward hotspot detection," IEEE Transactions on Automation Science and Engineering, vol. 16, no. 1, pp. 351-362, 2018.

[2] M. Ghahramani, M. Zhou, and G. Wang, "Urban sensing based on mobile phone data: approaches, applications, and challenges," IEEE/CAA Journal of Automatica Sinica, vol. 7, no. 3, pp. 627-637, 2020.

[3] T. Glenn and S. Monteith, "New measures of mental state and behavior based on data collected from sensors, smartphones, and the Internet," Current Psychiatry Reports, vol. 16, no. 12, p. 523, 2014.

[4] A. S. Shirazi, J. Clawson, Y. Hassanpour et al., "Already up? using mobile phones to track \& share sleep behavior," International Journal of Human-Computer Studies, vol. 71, no. 9, pp. 878-888, 2013.

[5] L. Chittaro and A. Vianello, "Computer-supported mindfulness: evaluation of a mobile thought distancing application on naive meditators," International Journal of Human-Computer Studies, vol. 72, no. 3, pp. 337-348, 2014.

[6] D. Ifenthaler, D. G. Sampson, and J. M. Spector, "Interactions between cognitive psychology, educational technology, and computing in the digital age," Technology, Knowledge and Learning, vol. 20, no. 2, pp. 129-131, 2015.

[7] K. Ahern and R. Le Brocque, "Methodological issues in the effects of attrition: simple solutions for social scientists," Field Methods, vol. 17, no. 1, pp. 53-69, 2005.

[8] M. M. Ramos-Álvarez, M. M. Moreno-Fernández, B. Valdés-Conroy, and A. Catena, "Criteria of the peer review process for publication of experimental and quasiexperimental research in Psychology: a guide for creating research papers," International Journal of Clinical and Health Psychology, vol. 8, no. 3, pp. 751-764, 2008. 
[9] A. Ardila, "On the evolutionary origins of executive functions," Brain and Cognition, vol. 68, no. 1, pp. 92-99, 2008.

[10] M. D. Lezak, D. B. Howieson, and D. W. Loring, Neuropsychological Assessment, Oxford University Press, New York, NY, USA, 1995.

[11] J. M. Baker, T. Martin, A. Aghababyan, A. Armaghanyan, and R. Gillam, "Cortical activations during a computer-based fraction learning game: preliminary results from a pilot study," Technology, Knowledge and Learning, vol. 20, no. 3, pp. 339-355, 2015.

[12] W. W. Graves, R. Desai, C. Humphries, M. S. Seidenberg, and J. R. Binder, "Neural systems for reading aloud: a multiparametric approach," Cerebral Cortex, vol. 20, no. 8, pp. 1799-1815, 2009.

[13] N. Miura, K. Iwata, J. Watanabe et al., "activation during reading aloud of long sentences: fMRI study," Neuroreport, vol. 14, no. 12, pp. 1563-1566, 2003.

[14] M. Watanabe and M. J. Taylor, "Is $2+2=4$ ? Meta-analyses of brain areas needed for numbers and calculations," Neuroimage, vol. 54, no. 3, pp. 2382-2393, 2011.

[15] R. Kawashima, M. Taira, K. Okita et al., "A functional MRI study of simple arithmetic-a comparison between children and adults," Cognitive Brain Research, vol. 18, no. 3, pp. 227-233, 2004.

[16] J. O. Watanabe and R. E. Ready, "Emotion and executive functioning: the effect of normal mood states on fluency tasks," Journal of Clinical and Experimental Neuropsychology, vol. 32, no. 3, pp. 225-230, 2010.

[17] A. J. Whitmer and I. H. Gotlib, "An attentional scope model of rumination," Psychological Bulletin, vol. 139, no. 5, pp. 1036-1061, 2013.

[18] H. Yang, S. Yang, and A. M. Isen, "Positive affect improves working memory: implications for controlled cognitive processing," Cognition \& Emotion, vol. 27, no. 3, pp. 474-482, 2013.

[19] K. J. Gilhooly and E. Fioratou, "Executive functions in insight versus non-insight problem solving: an individual differences approach," Thinking \& Reasoning, vol. 15, no. 4, pp. 355-376, 2009.

[20] E. K. Miller and J. D. Cohen, "An integrative theory of prefrontal cortex function," Annual Review of Neuroscience, vol. 24, no. 1, pp. 167-202, 2001.

[21] T. A. Niendam, A. R. Laird, K. L. Ray, Y. M. Dean, D. C. Glahn, and C. S. Carter, "Meta-analytic evidence for a superordinate cognitive control network subserving diverse executive functions," Cognitive, Affective, \& Behavioral Neuroscience, vol. 12, no. 2, pp. 241-268, 2012.

[22] J. Ward, The Student's Guide to Cognitive Neuroscience, Psychology Press, East Sussex, England, 2015.

[23] E. Angelakis, J. F. Lubar, and S. Stathopoulou, "Electroencephalographic peak alpha frequency correlates of cognitive traits," Neuroscience Letters, vol. 371, no. 1, pp. 60-63, 2004.

[24] S. Enriquez-Geppert, R. J. Huster, and C. S. Herrmann, "Boosting brain functions: improving executive functions with behavioral training, neurostimulation, and neurofeedback," International Journal of Psychophysiology, vol. 88, no. 1, pp. 1-16, 2013.

[25] R. M. Reitan, "Validity of the trail making test as an indicator of organic brain damage," Perceptual and Motor Skills, vol. 8, no. 3, pp. 271-276, 1958.

[26] C. Valiente-Barroso and E. García-García, "Executive function, adolescent development and mathematical competence: importance of quantitative and qualitative analysis in educational psychology," Procedia - Social and Behavioral Sciences, vol. 69, pp. 2193-2200, 2012.

[27] M. L. Turner and R. W. Engle, "Is working memory capacity task dependent?" Journal of Memory and Language, vol. 28, no. 2, pp. 127-154, 1989.

[28] H. L. St Clair-Thompson and S. E. Gathercole, "Executive functions and achievements in school: shifting, updating, inhibition, and working memory," Quarterly Journal of Experimental Psychology, vol. 59, no. 4, pp. 745-759, 2006.

[29] J. R. Best, P. H. Miller, and J. A. Naglieri, "Relations between executive function and academic achievement from ages 5 to 17 in a large, representative national sample," Learning and Individual Differences, vol. 21, no. 4, pp. 327-336, 2011.

[30] J. A. Naglieri and J. P. Das, Cognitive Assessment System, Riverside Publishing Co, Meadows, IL, USA, 1997.

[31] J. H. Gruzelier, "EEG-neurofeedback for optimising performance. I: a review of cognitive and affective outcome in healthy participants," Neuroscience \& Biobehavioral Reviews, vol. 44, pp. 124-141, 2014.

[32] D. Vernon, T. Egner, N. Cooper et al., "The effect of training distinct neurofeedback protocols on aspects of cognitive performance," International Journal of Psychophysiology, vol. 47, no. 1, pp. 75-85, 2003.

[33] T. Egner and J. H. Gruzelier, "EEG biofeedback of low beta band components: frequency-specific effects on variables of attention and event-related brain potentials," Clinical Neurophysiology, vol. 115, no. 1, pp. 131-139, 2004.

[34] C. Escolano, M. Aguilar, and J. Minguez, "EEG-based upper alpha neurofeedback training improves working memory performance," in Proceedings of the 2011 Annual International Conference of the IEEE Engineering in Medicine and Biology Society, pp. 2327-2330, Boston, MA, USA, September 2011.

[35] C. Escolano, M. Navarro-Gil, J. Garcia-Campayo, M. Congedo, D. De Ridder, and J. Minguez, "A controlled study on the cognitive effect of alpha neurofeedback training in patients with major depressive disorder," Frontiers in Behavioral Neuroscience, vol. 8, p. 296, 2014.

[36] J. H. Gruzelier, "EEG-neurofeedback for optimising performance. III: a review of methodological and theoretical considerations," Neuroscience \& Biobehavioral Reviews, vol. 44, pp. 159-182, 2014.

[37] R. Nouchi and R. Kawashima, "Improving cognitive function from children to old age: a systematic review of recent smart ageing intervention studies," Advances in Neuroscience, vol. 2014, Article ID 235479, 15 pages, 2014.

[38] R. Nouchi, Y. Taki, H. Takeuchi et al., "Brain training game improves executive functions and processing speed in the elderly: a randomized controlled trial," PloS One, vol. 7, no. 1, Article ID e29676, 2012.

[39] S. Uchida and R. Kawashima, "Reading and solving arithmetic problems improves cognitive functions of normal aged people: a randomized controlled study," Age, vol. 30, no. 1, pp. 21-29, 2008.

[40] G. M. Harari, N. D. Lane, R. Wang, B. S. Crosier, A. T. Campbell, and S. D. Gosling, "Using smartphones to collect behavioral data in psychological science," Perspectives on Psychological Science, vol. 11, no. 6, pp. 838-854, 2016.

[41] Y. S. Can, B. Arnrich, and C. Ersoy, "Stress detection in daily life scenarios using smart phones and wearable sensors: a survey," Journal of Biomedical Informatics, vol. 92, Article ID 103139, 2019.

[42] S. Rodgers, B. Maloney, B. Ploderer, and M. Brereton, "Managing stress, sleep and technologies: an exploratory study of Australian university students," in Proceedings of the 
2016 28th Australian Conference on Computer-Human Interaction, Launceston Tasmania Australia, November 2016.

[43] L. Chittaro and A. Vianello, "Mobile mindfulness and user's worry: a qualitative study of using a smartphone app for distancing from negative thoughts," Interacting with Computers, vol. 28, no. 6, pp. 695-717, 2016.

[44] G. M. Harari, S. R. Müller, M. S. Aung, and P. J. Rentfrow, "Smartphone sensing methods for studying behavior in everyday life," Current Opinion in Behavioral Sciences, vol. 18, pp. 83-90, 2017.

[45] J. J. Li and J. E. Lansford, "A smartphone-based ecological momentary assessment of parental behavioral consistency: associations with parental stress and child ADHD symptoms," Developmental Psychology, vol. 54, no. 6, pp. 1086-1098, 2018.

[46] N. Park and H. Lee, "Social implications of smartphone use: Korean college students' smartphone use and psychological well-being," Cyberpsychology, Behavior, and Social Networking, vol. 15, no. 9, pp. 491-497, 2012.

[47] S.-H. Yu, L.-S. Wang, H.-H. Chu et al., "A mobile mediation tool for improving interaction between depressed individuals and caregivers," Personal and Ubiquitous Computing, vol. 15, no. 7, pp. 695-706, 2011.

[48] J. Firth, J. Torous, J. Nicholas et al., “The efficacy of smartphonebased mental health interventions for depressive symptoms: a meta-analysis of randomized controlled trials," World Psychiatry, vol. 16, no. 3, pp. 287-298, 2017.

[49] J. H. L. Lui, D. K. Marcus, and C. T. Barry, "Evidence-based apps? A review of mental health mobile applications in a psychotherapy context," Professional Psychology: Research and Practice, vol. 48, no. 3, pp. 199-210, 2017.

[50] A. Howells, I. Ivtzan, and F. J. Eiroa-Orosa, "Putting the 'app' in happiness: a randomised controlled trial of a smartphone-based mindfulness intervention to enhance wellbeing," Journal of Happiness Studies, vol. 17, no. 1, pp. 163-185, 2016.

[51] R. Nouchi, Y. Taki, H. Takeuchi et al., "Brain training game boosts executive functions, working memory and processing speed in the young adults: a randomized controlled trial," $\mathrm{PloS}$ One, vol. 8, no. 2, Article ID e55518, 2013.

[52] R. Kawashima, K. Okita, R. Yamazaki et al., "Reading aloud and arithmetic calculation improve frontal function of people with dementia," The Journals of Gerontology Series A: Biological Sciences and Medical Sciences, vol. 60, no. 3, pp. 380-384, 2005.

[53] R. Nouchi, Y. Taki, H. Takeuchi, T. Nozawa, A. Sekiguchi, and R. Kawashima, "Reading aloud and solving simple arithmetic calculation intervention (learning therapy) improves inhibition, verbal episodic memory, focus attention and processing speed in healthy elderly people: evidence from a randomized controlled trial," Frontiers in Human Neuroscience, vol. 10, p. $217,2016$.

[54] A. Lomov, OpenShift and Cloud Foundry PaaS: High-Level Overview of Features and Architectures, Altoros, Pleasanton, CA, USA, 2014.

[55] S. H. Yu, C. Y. Tseng, and W. L. Lin, "A neurofeedback protocol for executive function to reduce depression and rumination: a controlled study," Clinical Psychopharmacology and Neuroscience, vol. 18, no. 3, p. 375, 2020.

[56] H. Marzbani, H. R. Marateb, and M. Mansourian, "Neurofeedback: a comprehensive review on system design, methodology and clinical applications," Basic and Clinical Neuroscience, vol. 7, no. 2, p. 143, 2016.

[57] M. J. Begemann, E. J. Florisse, R. Van Lutterveld, M. Kooyman, and I. E. Sommer, "Efficacy of EEG neurofeedback in psychiatry: a comprehensive overview and meta- analysis," Translational Brain Rhythmicity, vol. 1, pp. 19-29, 2016.

[58] R. D. Rogers and S. Monsell, "Costs of a predictible switch between simple cognitive tasks," Journal of Experimental Psychology: General, vol. 124, no. 2, pp. 207-231, 1995.

[59] S. Kéri, "Solitary minds and social capital: latent inhibition, general intellectual functions and social network size predict creative achievements," Psychology of Aesthetics, Creativity, and the Arts, vol. 5, no. 3, pp. 215-221, 2011.

[60] A. Miyake and N. P. Friedman, "The nature and organization of individual differences in executive functions," Current Directions in Psychological Science, vol. 21, no. 1, pp. 8-14, 2012.

[61] J. Bomyea and N. Amir, "The effect of an executive functioning training program on working memory capacity and intrusive thoughts," Cognitive Therapy and Research, vol. 35, no. 6, pp. 529-535, 2011.

[62] P. L. Rock, J. P. Roiser, W. J. Riedel, and A. D. Blackwell, "Cognitive impairment in depression: a systematic review and meta-analysis," Psychological Medicine, vol. 44, no. 10, pp. 2029-2040, 2014.

[63] Á. López-Martínez, S. Santiago-Ramajo, A. Caracuel, C. Valls-Serrano, M. J. Hornos, and M. J. Rodriguez-Fortiz, "Game of gifts purchase: computer-based training of executive functions for the elderly," in Proceedings of the 2011 IEEE 1st International Conference on Serious Games and Applications for Health (SeGAH), Braga, Portugal, November 2011.

[64] J. J. Summers, A. Wolf, S. Elder, M. Summers, K. Limbrick, and M. Tyler, "O4-07-04: cognitive training in older adults," Alzheimer's \& Dementia, vol. 6, no. 4, pp. S163-S164, 2010. 